\title{
Percepción ciudadana de los recursos fúngicos y potencial de micoalfabetización en Costa Rica
}

\author{
Citizen perception of fungal resources and mycoliteracy potential \\ in Costa Rica
}

\author{
Carlos Rojas ${ }^{1,2}$, Sergio Molina-Murillo1,3 \\ ${ }^{1}$ Instituto de Investigaciones en Ingeniería, Universidad de Costa Rica, San Pedro de Montes de Oca, 11501-Costa Rica. \\ ${ }^{2}$ Escuela de Ingeniería de Biosistemas, Universidad de Costa Rica, San Pedro de Montes de Oca, 11501-Costa Rica. \\ ${ }^{3}$ Escuela de Ciencias Ambientales, Universidad Nacional, Heredia, 30101-Costa Rica.
}

\section{RESUMEN}

Antecedentes: La integración entre información biológica y datos sobre la percepción ciudadana de los recursos naturales es clave para construir iniciativas de conservación biológica sensibles a las diferencias socioculturales.

Objetivos: Documentar las diferencias en la percepción sobre los recursos micológicos en la población general de Costa Rica a lo largo del territorio de este país.

Métodos: Se realizaron 1361 encuestas según un diseño con un 90 \% de confianza, en 11 ciudades de Costa Rica. Se analizaron las respuestas obtenidas sobre los hongos a partir de criterios sociodemográficos y regionales.

Resultados y conclusiones: Se encontró una asociación entre un mayor nivel de conocimiento y sensibilidad hacia los recursos fúngicos y un mayor nivel de educación. Esta asociación estuvo relacionada también con una mejor interpretación de la funcionalidad ecológica de los hongos. Los conocimientos generales sobre tipos percibidos de hongos disminuyeron en las regiones alejadas al Área Metropolitana y las zonas costeras del pacífico mostraron los niveles más bajos de conocimiento y apreciación por los hongos.

Palabras clave: biodiversidad, Centroamérica, educación, hongos, planeamiento

\section{ABSTRACT}

Background: The integration between biological information and data on citizen perception of natural resources is key for socioculturally-sensitive initiatives of biological conservation.

Objectives: To document perceptional differences on fungal resources in the general population of Costa Rica along its national territory.

Methods: A total of 1361 surveys were carried out in a design with a $90 \%$ of confidence. These were conducted in 11 cities of Costa Rica. Response patterns on fungal resources were analyzed according with sociodemographic and regional criteria. Results and conclusions: An association between higher knowledge on fungi was found in populations with higher educational level. This association influenced the public interpretation of ecological functionality of fungi. General knowledge on fungi decreased in regions farther away from the Metropolitan Areas and coastal cities along the pacific coast of Costa Rica showed the lowest levels of fungal knowledge and appreciation.

Keywords: biodiversity, Central America, education, fungi, planning

\section{ARTICLE HISTORY}

Received 22 June 2017 / Accepted 21 September 2018

On line 02 November 2018

\section{INTRODUCCIÓN}

Por muchos años, científicos de la naturaleza han estudiado los procesos de pérdida de hábitat y extinción de especies en áreas ricas en biodiversidad como Mesoamérica (Brooks et al., 2002). Al mismo tiempo, científicos sociales han estudiado el proceso de cambio en las construcciones culturales y han determinado

\section{CORRESPONDING AUTHOR \\ \4arlos Rojas Alvarado, carlos.rojasalvarado@ucr.ac.cr ORCID 0000-0002-7968-4833}

que para el año 2050, la estructura de pensamiento de muchas sociedades modernas será diferente a la de principios del siglo (Cohen, 2003). Dadas estas condiciones, es probable que muchos de los cambios paradigmáticos en relación con la naturaleza se traduzcan en pérdidas de conocimiento popular o de tradiciones caracterizadas por una alta interacción con recursos del planeta. Esto aumenta la probabilidad de poner en 
riesgo la funcionalidad de los ecosistemas (Naeem et al., 2012) por un mal manejo de estos ante tal nivel de desconexión. Por tanto, el estudio del nivel de apropiación que las sociedades humanas muestran hacia los recursos naturales resulta imperativo de documentar.

Dentro de este contexto, el poco peso sociopolítico que tienen un gran número de estudios de percepción acerca de los recursos naturales en la mayoría de sociedades se contradice con las políticas públicas de conservación que muchos países argumentan tener (Keulartz, 2009), y con los enfoques multidisciplinarios que se deberían de implementar para realizar conservación efectiva (Parker, 2011). Esto es importante debido a que la forma como las sociedades humanas perciben la naturaleza tiene un impacto sobre la interacción de estas con los biosistemas circundantes y el manejo de sus recursos naturales. Debido a que tal interacción no necesariamente puede ser medida en términos de indicadores objetivos (Diedrich y García-Buades, 2009; Molina-Murillo y Clifton, 2015), es imperativo que estudios sobre la percepción y el nivel de conocimiento sean llevados a cabo para poder evaluar efectivamente los cambios en enfoques sociales a través del tiempo y maximizar así, los esfuerzos de conservación al flexibilizar su implementación.

A pesar de ello, muchos esfuerzos para el desarrollo de marcos de trabajo conceptuales en conservación biológica siguen enfocándose en el estudio de los sistemas biológicos más que en la interacción funcional con poblaciones humanas. Excepciones claras en Latinoamérica incluyen los esfuerzos de Victor Manuel Toledo Manzur (2013) y Eckart Boegue (2015). Lo mencionado es importante debido a que el manejo exitoso de los recursos naturales es una tarea compleja que requiere del entendimiento del vínculo entre sistemas biológicos y sociopolíticos (Larson et al., 2013). Por ejemplo, el mejoramiento de las estrategias adaptativas al cambio climático en países en desarrollo requiere de la documentación del conocimiento popular y de las actitudes sociales en relación con los objetivos de desarrollo local (Molina-Murillo, 2013).

Localizada en el corazón de la zona de biodiversidad de Mesoamérica, Costa Rica se caracteriza por su riqueza natural (Joppa et al., 2011) y por el manejo exitoso que se le ha dado a los recursos naturales en las últimas décadas (Evans, 1999). Sin embargo, este país ha sido moderadamente estudiado en términos de diversidad fúngica y pobremente documentado en aspectos etnomicológicos (Molina-Murillo et al., 2015). En este territorio, la mayoría de los estudios biológicos y esfuerzos de conservación han sido diseñados para estudiar plantas y animales, a pesar del hecho de que los hongos comprenden uno de los grupos más diversos del planeta y a que cumplen un papel fundamental para el mantenimiento de los ecosistemas (Lange, 2010). Por ejemplo, para Costa Rica se estima que los hongos representan alrededor del $13 \%$ de la biodiversidad del país (Obando, 2007). Esta deficiencia en el estudio de un grupo de organismos tan diverso e importante en el planeta no es única de este país y está presente a lo largo de la región latinoamericana.

Por estas razones, y con el fin de documentar diferencias percepcionales en zonas seleccionadas del territorio costarricense en relación con los recursos fúngicos, el presente estudio ha sido diseñado con el objetivo de ofrecer un panorama general del nivel de alfabetización micológica (entendida como cualquier conocimiento sobre los hongos que tenga base científica) que tiene la población local. Si bien, el presente estudio es puntual en tiempo y espacio, su enfoque promueve una generación de datos sociales de interacción con elementos naturales que no necesariamente se tienen en otras zonas de la región latinoamericana, y por ello, se visualiza como un promotor de conservación integrada en el campo micológico. Lo anterior es esencial para desarrollar esfuerzos integrados de estudio, conservación y manejo de los hongos, y para optimizar el manejo y conservación de un grupo de organismos que facilitan la existencia de la naturaleza como se conoce.

\section{MATERIALES Y MÉTODOS}

El presente proyecto fue llevado a cabo desde la Unidad de Recursos Forestales del Instituto de Investigaciones en Ingeniería de la Universidad de Costa Rica durante los años 2016 y 2017. La metodología de trabajo se basó en Molina-Murillo et al. (2015) y consistió en la realización de una encuesta directa a participantes anónimos, seleccionados de forma sistemática, en diferentes zonas geográficas de Costa Rica. Todas las encuestas fueron realizadas de forma oral a participantes localizados en zonas públicas como parques, mercados y estaciones de autobús y las respuestas fueron transcritas por los encuestadores. El instrumento de registro utilizado incluyó variables de información so- 
ciodemográfica como edad, sexo y nivel de educación formal, y registró datos acerca de conocimientos generales de los hongos como la funcionalidad ecológica, los usos socioeconómicos y la percepción general sobre este grupo de organismos. Las preguntas analizadas han sido incluidas como secciones discretas en los resultados y se enfocaron en conocimientos ecológicos-funcionales y utilización de los hongos a nivel socioeconómico.

Para poder realizar el muestreo distribuido a lo largo del país, se seleccionaron 11 ciudades con población mayor a 10000 habitantes con base en su localización geográfica e importancia regional. Las ciudades dentro de la región Vertiente Caribe (R1) incluidas en este estudio fueron Ciudad Quesada (CQ) y Puerto Limón $(\mathrm{PL})$. Esta región presenta bosques húmedos y un clima húmedo y caliente con una temperatura media de 26 ${ }^{\circ} \mathrm{C}$ y una precipitación anual de $3000 \mathrm{~L} / \mathrm{m}^{2}$ por año. En la región Pacífico Sur (R2), con una temperatura media de $26{ }^{\circ} \mathrm{C}$, una precipitación anual de $4000 \mathrm{~L} / \mathrm{m}^{2}$ y bosques húmedos a muy húmedos, las ciudades escogidas fueron San Isidro de El General (SI) y Ciudad Neilly (CN). La zona de bosque seco o semiseco de Costa Rica, en el Pacífico Norte y Medio (R3), se caracteriza por una temperatura media de $28{ }^{\circ} \mathrm{C}$ y precipitación anual de $1500 \mathrm{~L} / \mathrm{m}^{2}$. Dentro de esta zona las ciudades escogidas fueron Liberia (LI), Nicoya (NI) y Puntarenas (PU). Finalmente, San Ramón (SR), Paraíso (PA), San José (SJ) y San Pedro de Montes de Oca (SP) fueron escogidas dentro de la región Central (R4, ver agrupamiento en Figura 1), que se caracteriza por bosques premontanos húmedos con una temperatura media de $22{ }^{\circ} \mathrm{C}$ y precipitación anual de $2000 \mathrm{~L} / \mathrm{m}^{2}$. En el caso de ésta última, correspondiente al Área Metropolitana de Costa Rica, la selección se hizo también para evaluar de forma interna, diferencias asociadas a patrones urbanos o históricos en esta región (p.ej. el desarrollo urbano y la oferta académica se han extendido de este a oeste en esta región).

En cada ciudad escogida se realizaron entre 120 y 130 encuestas para un total de 1361 en todo el país. El anterior diseño ofreció un $5 \%$ de margen de error y un 90 $\%$ de confianza. En todos los casos, las respuestas fueron estandarizadas, nominalizadas y analizadas de forma individual en primera instancia. Para el análisis por regiones y ciudades, se creó un índice de conocimiento a través del cual se normalizaron en una escala de 1 a 100 los valores de tres preguntas relacionadas con el conocimiento percibido por los participantes sobre hongos (i.e., número de tipos de hongos identificados, número de afirmaciones funcionales seleccionadas, y afirmación correcta de que los hongos pueden ser de beneficio para las plantas). La idea de este índice es evaluar de forma integrada y minimizando errores de interpretación, las similitudes o diferencias en los patrones de respuesta entre regiones, para poder ofrecer una comparación más objetiva en el análisis llevado a cabo.

Aquellos casos con valores de desviación a nivel de ciudad o región fueron analizados a partir de pruebas de Chi Cuadrado de bondad de ajuste utilizando valores de hipótesis determinados por la distribución general. Para evaluar diferencias en la distribución de respuestas por sexo, edad o escolaridad se utilizaron pruebas de Chi Cuadrado de Pearson. En aquellos casos con desviaciones significativas a los valores promedio de ciudad o región, se cuantificaron las variables de edad y escolaridad en términos de edad promedio por grupo etario o años promedio de estudio para evaluar correlaciones ( $r$ de Pearson) con las respuestas ofrecidas. Todas las categorías de hongos mostradas son del tipo "etic"; es decir, construidas a posteriori para efectos de análisis, por los investigadores.

\section{RESULTADOS}

Del total de personas entrevistadas, un $53 \%$ fueron hombres y un $47 \%$ mujeres. Un $42 \%$ de estas personas tuvieron edades entre los 16 y los 25 años, seguidos de los intervalos de 26 a 35 años y 36 a 45 años, representados con el $18 \%$ y el $11 \%$ de los participantes. En términos de escolaridad, los grupos mejor representados fueron el de educación secundaria incompleta con un $29 \%$ de los registros, el de secundaria completa y el de universidad incompleta, ambos con un $20 \%$ de todos los participantes. Los valores generales de edad y escolaridad no fueron diferentes a lo reportado para Costa Rica en la Encuesta Nacional a Hogares (INEC) del año 2017 ( $P=0.08$, en ambos casos).

Con respecto a los valores de referencia del total de participantes evaluados en el estudio completo, únicamente las ciudades de San Ramón y San José mostraron desviaciones significativas en la división de participantes por sexo. En estos casos, se entrevistaron marginalmente más hombres que mujeres en San José $(P=0.03)$ y viceversa en San Ramón $(P=0.04)$. Ninguna 
diferencia fue encontrada en la distribución de edades por ciudad. En relación con escolaridad, únicamente los participantes de San Pedro de Montes de Oca mostraron un patrón significativamente más alto que el resto de las poblaciones estudiadas (60 \% con educación universitaria, $P<0.001$ ).

\section{¿Sabe usted lo que es un hongo?}

La idea de esta pregunta era documentar el grado de apropiamiento conceptual de las poblaciones estudiadas; es decir, si creen saber lo que es un hongo. El 90 $\%$ de los participantes dijo conocer lo que es un hongo y a pesar de que la distribución general de respuestas se mantuvo estable en todas las ciudades, en Liberia y San Isidro de El General los participantes respondieron positivamente en menor grado (alrededor de $80 \%$, $P=0.004$ en ambos casos). De forma similar, en Puerto Limón y San Pedro de Montes de Oca los participantes mostraron el comportamiento opuesto con alrededor de un $96 \%$ de respuestas positivas $(P=0.04)$. Los valores más bajos de respuestas positivas estuvieron asociados con las ciudades en zonas secas de la costa pacífica del país, mientras que en las ciudades del centro y Puerto Limón se encontraron valores más altos que la media general.

Los valores de respuesta asociados con esta pregunta no mostraron diferencias significativas por edad, pero si por escolaridad $(P<0.001)$. En este último caso, se observó que a mayor escolaridad los participantes respondieron la pregunta de forma positiva con mayor frecuencia, con una consecuente disminución en el patrón de respuestas negativas. La correlación entre estas dos variables fue alta $\left(R^{2}=0.86\right)$, aun al quitar a la población de San Pedro de Montes de Oca del análisis $\left(R^{2}=0.90\right)$, lo que sugiere un efecto importante de la educación general sobre los conocimientos básicos de los hongos y la naturaleza.

¿Ha escuchado usted acerca de tipos de hongos?

Cinco categorías de tipos de hongos que incluyeron comestibles, alucinógenos, medicinales, patógenos y otros (i.e. aquellos usados en procesos industriales o aplicaciones agrícolas), fueron dadas a los participantes y se les pidió que respondieran si habían escuchado de hongos en la categoría respectiva. La idea de esta pregunta ha sido documentar conocimientos generales. Las respuestas mostraron que el $91 \%$ de los participantes respondió positivamente haber escucha- do de hongos comestibles, el $70 \%$ de hongos alucinógenos, el $61 \%$ de hongos medicinales, el $41 \%$ de hongos patógenos y el $7 \%$ de otros tipos.

Al analizar los resultados de los análisis estadísticos descriptivos realizados, las desviaciones de los datos por ciudad con respecto a los valores generales para el país, se observó que los participantes en San Pedro de Montes de Oca respondieron positivamente a una mayor cantidad de grupos de hongos que el resto de los participantes (Figura 1). En contraste, los participantes en la mayoría de las ciudades fuera del Área Metropolitana dijeron haber escuchado de un menor número de tipos o categorías de hongos y la distribución de respuestas en estas zonas mostró desviaciones negativas con respecto a la media nacional. Dentro de este último grupo, las respuestas registradas en Nicoya registraron los valores promedio más bajos (Figura 2). La menor variación interciudades se dio para los hongos comestibles, seguida de medicinales, en contraste con los alucinógenos y patógenos que registraron mayores variaciones (Figura 1).

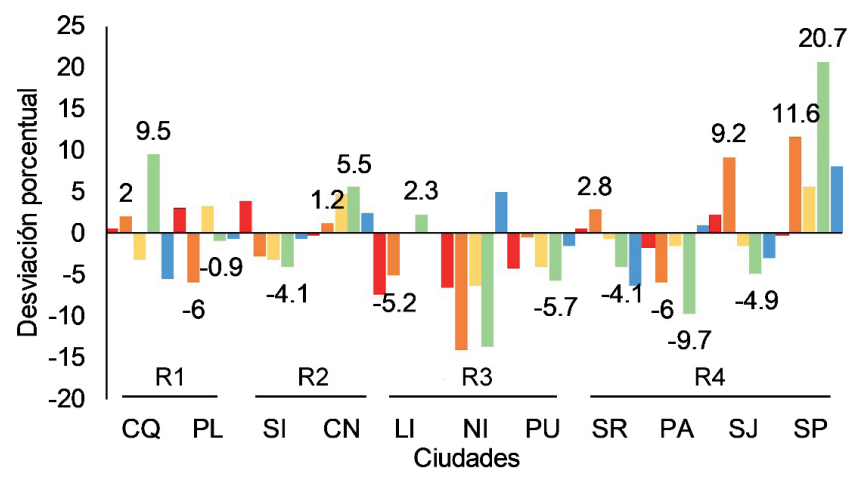

a Comestibles $\square$ Alucinógenos $\square$ Medicinales $=$ Patógenos $\approx$ Otros

$\mathrm{CQ}=$ Ciudad Quesada, PL=Puerto Limón, $\mathrm{SI}=$ San Isidro, CN=Ciudad Neilly, LI=Liberia, NI=Nicoya, PU=Puntarenas, SR=San Ramón, $\mathrm{PA}=$ Paraíso, $\mathrm{SJ}=$ San José, $\mathrm{SP}=$ San Pedro.

FIGURA 1. Desviaciones porcentuales con respecto a la media general para las diferentes ciudades y regiones a partir de las distintas categorías de tipos de hongos conocidos por los participantes del presente estudio.

Desviaciones positivas indican un mayor porcentaje de respuestas positivas y desviaciones negativas el patrón opuesto. El tono más oscuro y el tamaño más grande de los marcadores indican desviaciones positivas promedio más grandes (Figura 2). 


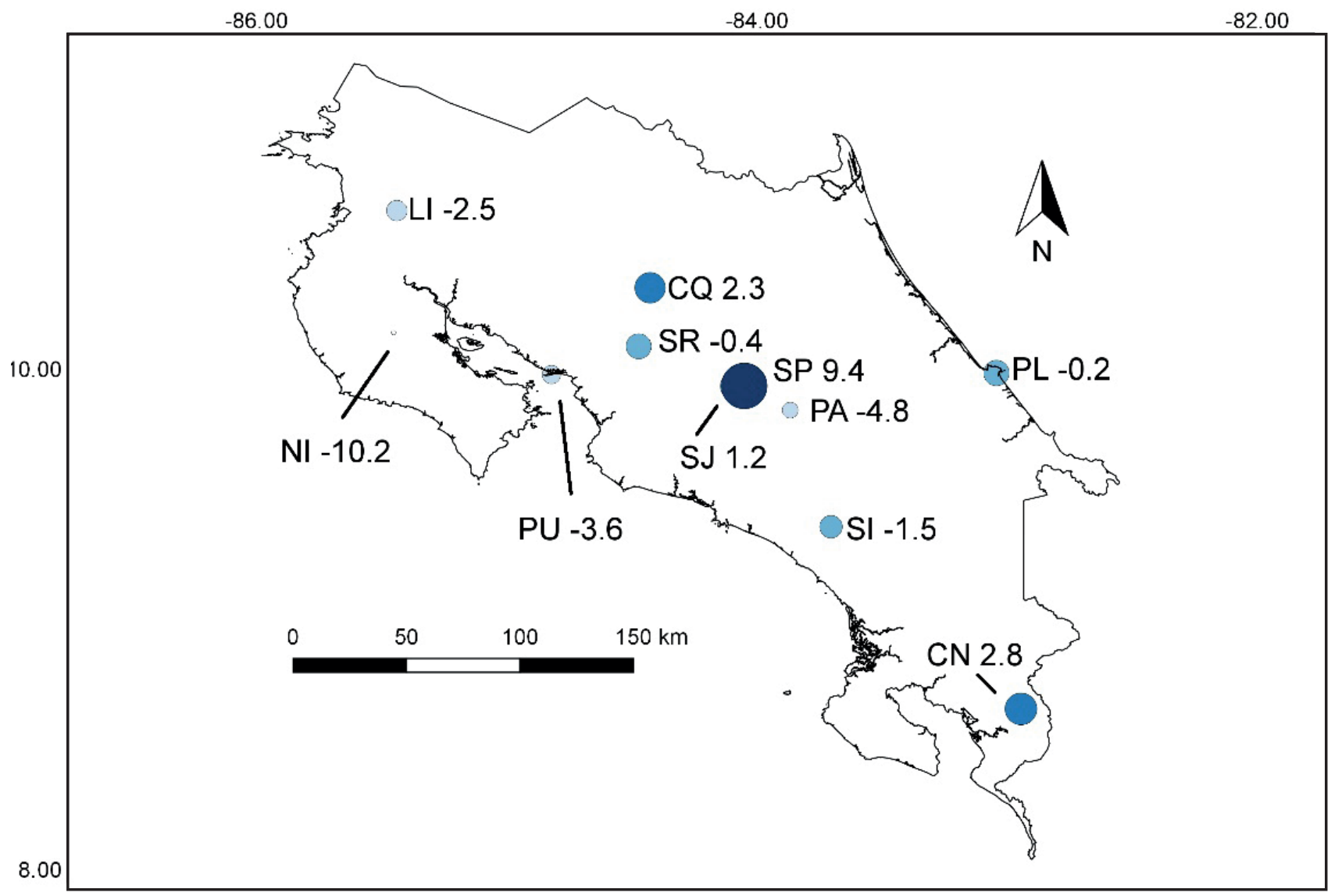

Figura 2. Mapa de Costa Rica mostrando los valores promedio, según ciudad, de las desviaciones porcentuales asociadas con las respuestas de los tipos de hongos conocidos.

¿Cuál grupo de organismos prefiere entre animales, plantas y hongos?

A los participantes se les pidió responder a la anterior pregunta colocando los tres grupos de organismos en orden de preferencia con el objetivo de observar el valor que se le da a cada uno de ellos. De forma general, un $56 \%$ de los participantes dijo preferir los animales, un $37 \%$ las plantas y un $7 \%$ los hongos. Sin embargo, se encontraron diferencias en la distribución de respuestas por sexo $(P=0.003)$ debido a que los hombres mostraron preferencia por el orden animales-plantas-hongos mientras que las mujeres mostraron preferencia por el orden plantas-animales-hongos. Con excepción de la distribución de respuestas en Puntarenas y San Pedro de Montes de Oca, ninguna ciudad mostró diferencias significativas con los valores generales obtenidos. Sin embargo, es interesante observar que, para las dos ciudades mencionadas, las diferencias se dieron por un menor número de participantes que el promedio, escogiendo los hongos como su grupo de preferencia $(P=0.03$ y $P<0.001$, respecti- vamente). Estas diferencias se mantuvieron cuando se comparó únicamente San Pedro de Montes de Oca con las otras ciudades dentro del Área Metropolitana $(\mathrm{P}=0.006)$.

De forma interesante, ninguna diferencia se encontró al analizar los valores de respuesta por escolaridad de los participantes, pero sí se observó por edad $(P=0.002)$, al ser las plantas preferidas y los hongos menos seleccionados a mayor edad. Estas últimas relaciones fueron tan pronunciadas, que se observaron correlaciones fuertes entre la edad promedio del grupo de edad evaluado y las respuestas ofrecidas $\left(R^{2}=0.85\right.$ para edad-preferencia por plantas y $R^{2}=0.78$ para edad-no preferencia por hongos).

\section{¿Cuál es el uso más importante de los hongos?}

La idea de esta pregunta ha sido documentar la percepción popular del uso de los hongos que ha sido percibido como el más importante. La distribución general de respuestas a esta pregunta mostró que el 44 $\%$ de los participantes asoció el uso más importante de 
los hongos con algún criterio de alimentación, el $26 \%$ con medicina, el $7 \%$ con un rol ecológico y un $1 \%$ con uso recreativo alucinógeno. Sin embargo, de forma interesante, las desviaciones significativas a estos valores fueron observadas primordialmente en ciudades fuera del Área Metropolitana.

Por ejemplo, en Ciudad Neilly un porcentaje mayor de participantes asoció el uso más importante con el criterio recreativo alucinógeno y un menor porcentaje con el rol ecológico $(P=0.01)$. En Liberia y Nicoya, los participantes respondieron con menor frecuencia los criterios alimenticio y ecológico y en el caso de la primera, con mayor frecuencia el uso recreacional $(P=0.01$ y $P=0.008$, respectivamente). En Puerto Limón, se observó que los participantes respondieron más veces el criterio alimenticio y en menor grado el rol ecológico $(P=0.006)$. La excepción fue San Pedro de Montes de Oca en donde un mayor número de participantes que el promedio respondió el criterio ecológico $(P=0.001)$. Esta diferencia también se observó con respecto a las otras ciudades dentro del Área Metropolitana $(P<0.001)$.

De forma interesante, para la distribución de respuestas asociadas con esta pregunta no se observaron patrones significativamente diferentes por edad, pero si se observaron por escolaridad, en una categoría de respuesta. En este último caso, se observó que el número de respuestas asociadas con la categoría de medicina aumentó con la escolaridad ( $P=0.001)$, aun al quitar a la población de San Pedro de Montes de Oca $(P=0.001)$. La correlación entre los años de estudio y la frecuencia de respuestas en esta categoría fue alta $\left(R^{2}=0.86\right)$. Como referencia, la correlación de años de estudio con la frecuencia de respuestas asociadas al rol ecológico fue intermedia $\left(R^{2}=0.47\right)$.

\section{Afirmaciones funcionales}

A los participantes se les solicitó contestar cuáles de cuatro afirmaciones sobre la funcionalidad de los hongos en la vida cotidiana de las sociedades eran ciertas. La idea de la pregunta ha sido observar el nivel de integración conceptual que los participantes han tenido en relación con las funciones ecológicas de los hongos. Las cuatro afirmaciones eran verdaderas y correspondieron con: 1) muchos hongos son patógenos de plantas y animales, 2) los hongos son importantes para el equilibrio de los ecosistemas, 3) los hongos se usan para producir medicamentos y 4) muchos hongos presentan asociaciones con plantas. En términos generales, la frecuencia asociada con todas las respuestas y con el número de respuestas contestadas fue cercana a un $25 \%$, es decir, no diferente a una distribución aleatoria.

Al analizar los patrones de respuesta por ciudad (Figura 3), se pudo observar que los participantes en San Pedro de Montes de Oca contestaron positivamente en mayor grado un número de tres y cuatro respuestas (37\% y $31 \%$, respectivamente, $\mathrm{P}=0.001$ ). Un patrón similar se observó para Ciudad Quesada $(P=0.01)$. De forma opuesta, los participantes de Nicoya contestaron con mayor frecuencia un número de una o dos respuestas (40\% y $27 \%$, respectivamente, $\mathrm{P}=0.001$ ). Dentro del Área Metropolitana, San José y San Pedro de Montes de Oca mostraron tres y cuatro respuestas con mayor frecuencia diferente que San Ramón y Paraíso $(P=0.001)$.

A pesar de lo anterior, las frecuencias asociadas con el número de respuestas tuvieron diferencias por grupo de edad $(P=0.001)$ y años de escolaridad $(P=0.001$ y $\mathrm{P}=0.002$ excluyendo a San Pedro de Montes de Oca). En el primer caso, el número de participantes respondiendo positivamente a una o dos respuestas disminuyó significativamente con mayor edad, lo que permitió que el número asociado con tres o cuatro respuestas aumentara en el mismo sentido. La correlación entre ambos patrones fue alta $\left(R^{2}=0.83\right)$ mostrando un cambio en el número de respuestas a mayor edad. Con respecto a la escolaridad, también se observó una correlación alta $\left(R^{2}=0.95\right)$ entre los años de estudio y un mayor número de respuestas ofrecidas, sugiriendo un efecto importante de la educación sobre la información manejada por los participantes del estudio.

\section{Nivel de conocimiento general sobre hongos}

El nivel de conocimiento general sobre hongos determinado a través del Índice de conocimiento fue relativamente bajo (62) con diferencias significativas a nivel de ciudades (Wilcoxon $Z=71.83$, g.l. $=10, P<0.001$ ) y regiones (Wilcoxon $Z=53.42$ g.l. $=3, P<0.001$ ). Específicamente la ciudad de San Pedro fue la que mostró el valor promedio más alto (73) con un valor significativamente distinto al resto de las ciudades excepto por Ciudad Quesada con 66 (Figura 4). A nivel de regiones, la Central y la Vertiente Caribe mostraron los valores más altos (73 y 72 respectivamente) y la región Pacífico Norte y Medio fue la que mostró significativamente un valor distinto al resto (63). 


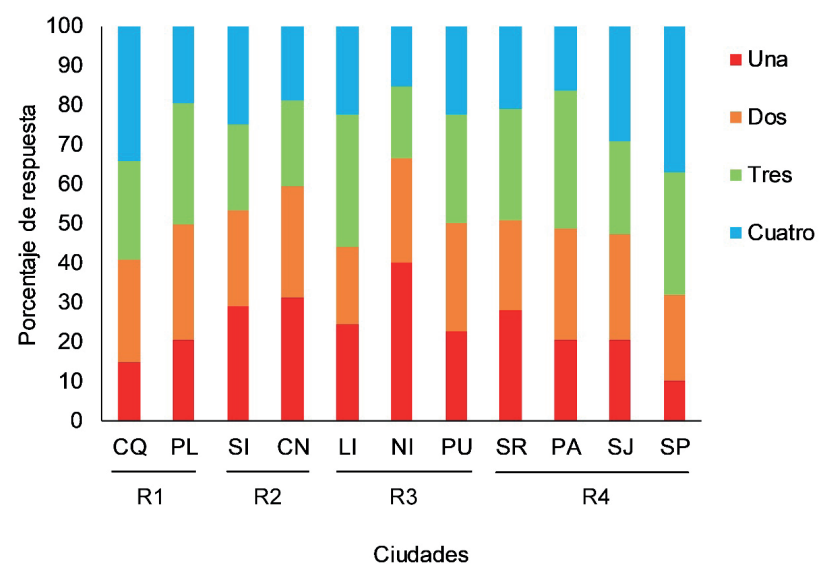

Figura 3. Porcentajes asociados con el número de respuestas dadas por los participantes en cada ciudad y región estudiada ante cuatro afirmaciones funcionales de los hongos en la vida cotidiana.

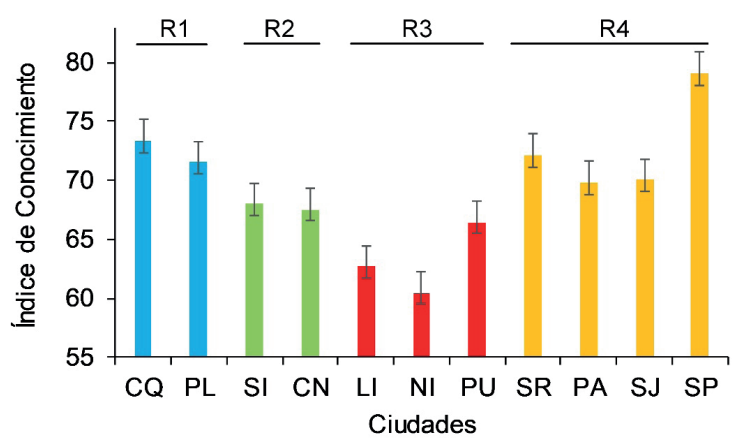

R1=Vertiente Caribe, R2=Pacífico Sur, R3=Pacífico Norte y Medio, R4=Región Central.

Figura 4. Índice de conocimiento por ciudades de estudio y regiones, con indicación del error estándar.

\section{DISCUSIÓN}

En términos generales, la distribución observada de respuestas refleja una baja apreciación de las poblaciones costarricenses estudiadas por el reino de los hongos. Esto no necesariamente se ve reflejado en el nivel de conocimientos, que es variable dependiendo de la zona geográfica, pero señala que, para la mayoría de los participantes de este estudio, los hongos podrían tener un valor sociocultural más bajo que otros elementos de la naturaleza. La diferencia anterior es clave para evaluar si en Costa Rica podría existir una conexión emocional baja con los hongos, ya que sin ella es muy complejo que una valoración efectiva de elementos naturales se lleve a cabo (García Retana, 2012).

Si bien, lo anterior no es sorprendente en el marco de estudios previos (Molina-Murillo et al., 2015), los resultados de este estudio muestran que la carencia de co- nocimientos sobre los hongos en Costa Rica está asociada con las zonas geográficas más áridas y calientes (bosque seco y bosque transicional del pacífico norte y central). Lo anterior es interesante, debido a que son precisamente estas zonas, donde existe influencia histórico-cultural mesoamericana, presumiblemente micofílica, y al hecho de que la productividad fúngica no es necesariamente baja (Rojas et al., 2017). A pesar de lo anterior, existe evidencia similar de que en otras zonas bajas y secas dentro de Mesoamérica, las poblaciones son menos micófilas que en zonas con alto porcentaje de hongos ectomicorrícicos, como los bosques de alturas (Ruan-Soto et al., 2004). De esta forma, los datos ofrecidos en este estudio sugieren que no es tal influencia cultural sino la educación formal normalizada a nivel territorial, el aspecto que podría estar asociado con la distribución de los datos. Por ejemplo, en Ciudad Quesada, centro de desarrollo semiurbano del norte del país y con escolaridad media alta, los niveles de conocimiento general mostraron valores similares a ciudades en la sección urbana central del país. En contraste, las zonas semiurbanas en donde los participantes tuvieron baja escolaridad, como Nicoya, Liberia y Pérez Zeledón, los patrones de respuestas fueron diferentes.

A pesar de lo anterior, resulta interesante documentar que, para la población costarricense, el uso más importante de los hongos puede estar asociado con un criterio alimenticio. Con excepción de San Pedro de Montes de Oca, en donde los participantes mostraron alta educación formal, en la mayoría de las ciudades no se documentaron varias categorías de uso con frecuencia similar. Desde el punto de vista de seguridad alimentaria y para efectos de manejo de los recursos fúngicos a nivel nacional, lo anterior no es trivial (Rodríguez y Sanders, 2015). Si bien, los datos ofrecidos en este estudio señalan que la mayoría de los participantes no asociaron a los hongos de forma frecuente con otros usos (algo ya documentado en Molina-Murillo et al., 2015), también demuestra que, para el caso de Costa Rica, existe al menos un punto de entrada sociocultural para fortalecer la apreciación hacia este grupo de organismos.

Lo anterior quizás no proviene de elementos histórico-culturales asociados con el territorio costarricense, primordialmente localizado en la zona antropológica de influencia intermedia. Presumiblemente está asociado con la frecuencia de consumo de elementos 
dietarios importados, como la pizza con champiñones, en las últimas décadas. Un patrón similar ha sido comunicado previamente para México (Mayett et al., 2008). Sin embargo, en el caso costarricense, esta asociación ha permitido un desarrollo de la micofagia en zonas urbanas, con una consecuente valoración del uso alimenticio de los champiñones y otros macrohongos comúnmente utilizados (p.e. hongos ostra y shiitake).

Es interesante documentar que, para la mayoría de los consumidores de este alimento en Costa Rica, los hongos provienen de una lata con origen industrial y no necesariamente de un sistema biológico debido a que raramente se usan hongos frescos en la preparación de pizza. Esta diferencia en el origen del producto disminuye el poder de interacción entre el usuario, el alimento y la fuente natural. De forma contrastante, en otros territorios centroamericanos como Guatemala, Honduras o El Salvador, la venta de hongos recolectados en el campo se observa en mercados tradicionales (Boa, 2004) y también se mantienen recetas tradicionales de consumo de hongos.

Independientemente de lo anterior, los resultados del presente estudio parecen señalar que, en el caso de Costa Rica, existe homogeneidad en la percepción de los recursos fúngicos, influenciada de forma primaria por el aspecto educativo. De esta forma, el poder de la micoalfabetización a nivel nacional no puede ser ignorado. Si bien los resultados muestran que todavía existe un nivel grande de desconocimiento formal (académico) del rol de los hongos (a pesar de que la función ecológica de los mismos fue documentada con alta frecuencia en la ciudad con mayor nivel educativo), existe un potencial grande de educación biológica en la sociedad costarricense, como también ha sido documentado en otras latitudes (Oliveira-Silva y Santos-Baptista, 2017). Lo anterior en parte se debe al alto valor que esta sociedad le confiere a la educación formal. Por ejemplo, en un estudio realizado en una escuela pública semiurbana (datos no mostrados), se ha documentado un incremento significativo en los conocimientos sobre el rol de los hongos en procesos de degradación y fertilización biológica tras incorporar proyectos de investigación con la comunidad escolar y trabajar con los niños por un año. De esta forma, como ha sido propuesto por Boney et al. (2014) una estrategia de micoalfabetización podría surgir a partir proyectos de ciencia ciudadana, que permitan que las poblaciones adquieran conocimientos de su propio interés a su propio ritmo. La ventaja de la promoción de este tipo de estrategia radica en el hecho de que los hongos, son un grupo biológico que se presta para promover la biofilia (Simaika y Samways, 2010). En el caso de Costa Rica, la promoción reciente de estudios de dinámica fúngica dentro de un marco de cambio climático y servicios del ecosistema (Hirons et al., 2016; Rojas y Valverde, 2019) han llevado información micológica a poblaciones humanas en zonas semiurbanas. Estos esfuerzos actuales podrían utilizarse para evaluar si la actividad científica formal puede tener un efecto sobre el interés ciudadano por el tema de los hongos y para analizar si estrategias como la propuesta acá pueden ser implementadas efectivamente, con el fin último de promover la conservación forestal (Suzart de Albuquerque et al., 2015).

De cualquier forma, un aspecto clave adicional documentado con este proyecto fue el hecho de que, en el uso del idioma español en Costa Rica, existen pocas palabras para referirse a los miembros del reino Fungi. Sería interesante explorar con mayor detalle y con un enfoque etimológico, tal utilización de palabras a nivel regional, con el objetivo de evidenciar la carga cultural asociada con los hongos a partir del uso del idioma. Finalmente, en este estudio se evidenció que los criterios de manejo de la naturaleza y política pública de conservación deberían de estar vinculados de alguna forma con las diferencias regionales de percepción, que aun en un país pequeño como Costa Rica se han podido documentar para un grupo específico de organismos.

\section{AGRADECIMIENTOS}

Este estudio ha sido financiado por la Vicerrectoría de Investigación de la Universidad de Costa Rica a través de los proyectos 731-B4900, 731-B8-033 y 731-B5-057. Se extiende un agradecimiento a Stephanie Somerville, Randall Valverde y Pedro Rojas por ayuda en el campo durante el proceso de toma de datos. Finalmente agradecemos a dos revisores anónimos por sus acertados y constructivos comentarios.

\section{LITERATURA CITADA}

Boa, E., 2004. Wild edible fungi: a global overview of their use and importance to people. Non-Wood Forest Products 17. FAO, Rome.

Boegue, E., 2015. Em direção a uma antropologia ambiental para a apropriação do patrimônio biocultural dos povos indígenas na 
América Latina. Desenvolvimiento e Meio Ambiente 35: 101120. Doi: http://dx.doi.org/10.5380/dma.v35i0.43906

Boney, R., J.L. Shirk, T.B. Phillips, A. Wiggins, H.L. Ballard, A.J. Miller-Rushing, J.K. Parrish, 2014. Next steps for citizen science. Science 343(6178): 1436-1437. Doi: http://dx.doi.org/10.1126/ science. 1251554

Brooks, T.M., R.A. Mittermeier, C.G. Mittermeier, G.A.B. Da Fonseca, A.B. Rylands, R.A. Konstant, R. Flick, J. Pilgrim, S. Oldfield, G. Magin, C. Hilton-Taylor, 2002. Habitat loss and extinction in the hotspots of biodiversity. Conservation Biology 16: 909-923. Doi: https://doi.org/10.1046/j.1523-1739.2002.00530.x

Cohen, J.E., 2003. Human population: The next half century. Science 302: 1172-1175. Doi: https://doi.org/10.1126/science.1088665

Diedrich, A., E. García Buades, 2009. Local perceptions of tourism as indicators of tourism destination decline. Tourism Management 30: 512-521. Doi: https://doi.org/10.1016/j.tourman.2008.10.009

Evans, S., 1999. The green republic. The University of Texas Press, Austin, Texas.

García Retana, J.A., 2012. La educación emocional, su importancia en el proceso de aprendizaje. Revista Educación 36(1): 1-24. Doi: https://doi.org/10.15517/revedu.v36i1.455

Hirons, M., C. Comberti, R. Dunford, 2016. Valuing cultural ecosystem services. Annual Review of Environment and Resources 41: 545-574. Doi: https://doi.org/10.1146/annurev-environ-110615-085831

Joppa, L.N., D.L. Roberts, N. Myers, E.L. Pimm, 2011. Biodiversity hotspots house most undiscovered plant species. PNAS 108:13171-13176. Doi: https://doi.org/10.1073/ pnas. 1109389108

Keulartz, J., 2009. European nature conservation and restoration policy-problems and perspectives. Restoration Ecology 17(4): 446450. Doi: https://doi.org/10.1111/j.1526-100X.2009.00566.x

Lange, L., 2010. The importance of fungi for a more sustainable future on our planet. Fungal Biology Reviews 24: 90-92. Doi: https:// doi.org/10.1016/j.fbr.2010.12.002

Larson, S., D.M. De Freitas, C.C. Hicks, 2013. Sense of place as a determinant of people's attitudes towards the environment: Implications for natural resources management and planning in the Great Barrier Reef, Australia. Journal of Environmental Management 117: 226-234. Doi: https://doi.org/10.1016/j.jenvman.2012.11.035

Mayett, Y., D. Martinez-Carrera, M. Sinchez, A. Macías, A. Moraaf, A. Estrada-Torres, 2008. Consumption trends of edible mushrooms in developing countries: The case of Mexico. Journal of International Food \& Agribusiness Marketing 18(1-2): 151-176. Doi: https://doi.org/10.1300/J047v18n01_08

Molina Murillo, S.A., 2013. Urban population knowledge of climate change in Costa Rica and Nicaragua. Revista Latinoamericana de Desarrollo Económico 19: 55-75.
Molina Murillo, S.A., J.T. Clifton, 2015. Perceptions of institutions and development in rural Costa Rica. Journal of Rural and Community Development 9(4): 80-101.

Molina-Murillo, S.A., C. Rojas, S. Somerville, R.G. Doss, B. Lemus, 2015. What do you think of fungi? Perceptions by "educated" groups in Honduras and Costa Rica. Brenesia 83-84: 30-36.

Naeem, S., J.E. Duffy, E. Zavaleta, 2012. The functions of biological diversity in an age of extinction. Science 336: 1401-1406. Doi: https://doi.org/10.1126/science.1215855

Obando, V., 2007. Biodiversidad de Costa Rica en cifras. INBio, Santo Domingo de Heredia.

Oliveira-Silva, E.A., G.C. Santos-Baptista, 2017. Un recurso acerca de los hongos para el diálogo intercultural en la enseñanza de biología. Revista Góndola, Enseñanza y Aprendizaje de las Ciencias 12(2): 142-157. Doi: https://doi. org/10.14483/23464712.11493

Parker, J.S., 2011. John Schelhas and Max J. Pfeffer: Saving forests, saving people? Environmental conservation in Central America. Agriculture and Human Values 28: 289-290. Doi: https://doi. org/10.1007/s10460-011-9313-2

Rodríguez, A., I.R. Sanders, 2015. The role of community and population ecology in applying mycorrhizal fungi for improved food security. The ISME Journal 9: 1053-1061. Doi: https://doi. org/10.1038/ismej.2014.207

Rojas, C., R. Valverde, R. Morales, 2017. Functional variability of macrofungal populations in four different forest types of Costa Rica. Mycosphere 8(9): 1288-1296. Doi: https://doi.org/10.5943/ mycosphere/8/9/3

Rojas, C., R. Valverde, 2019. Towards an integrated approach of conducting fungal research in Costa Rica. Uniciencia 33: 101-117. Doi: http://dx.doi.org/10.15359/ru.33-1.8

Ruan-Soto, F., R. Garibay-Orijel, J. Cifuentes, 2004. Conocimiento micológico tradicional en la planicie costera del Golfo de México. Revista Mexicana de Micología 19: 57-70.

Simaika, J.P., M.J. Sanways, 2020. Biophilia as a universal ethic for conserving biodiversity. Conservation Biology 24: 903-906. Doi: https://doi.org/10.1111/j.1523-1739.2010.01485.x

Suzart de Albuquerque, F., B. Benito, P. Beier, M.J. Assunção-Albuquerque, L. Cayuela, 2015. Supporting underrepresented forests in Mesoamerica. Natureza and Conservação 13(2): 152-158. Doi: https://doi.org/10.1016/j.ncon.2015.02.001

Toledo Manzur, V.M., 2013. Community conservation and ethnoecology: The three dimensions of local-level biodiversity maintenance. In: Porter-Bolland, L., I. Ruiz-Mallén, C. Camacho-Benavides, S. McCandless (eds.), Community Action for Conservation. Springer, New York. Doi: https://doi.org/10.1007/978-1-46147956-7_2 